\title{
Factores asociados a la contaminación con Campylobacter spp. termotolerante en pollos de engorde, en tres niveles de la cadena avícola, para consumo humano en Costa Rica.
}

\section{Factors associated to thermotolerant Campylobacter spp. contamination of broilers in three levels of the poultry chain for human consumption in Costa Rica}

\section{Leana Zumbado Gutiérrez ${ }^{1} \bowtie$, Juan José Romero Zúñiga²}

1 Cátedra de Inspección e Higiene de Alimentos, Escuela de Medicina Veterinaria, Universidad Nacional, Heredia, Costa Rica. Apdo. postal: 304-3000 Heredia. Teléfono: (506) 25624565. leana.zumbado.gutierrez@una.cr

2 Cátedra de Salud de Hato y Control de la Producción, Escuela de Medicina Veterinaria, Universidad Nacional, Heredia, Costa Rica. juan.romero.zuniga@una.cr

Recibido: 24 de Junio de 2016. Corregido: 19 de Octubre de 2016. Aceptado: 20 de Octubre de 2016.

Resumen. Campylobacter spp. es una bacteria destacada por ser causa frecuente de infecciones transmitidas por alimentos, tanto en países desarrollados como en vías de desarrollo. Se realizó un estudio transversal, entre marzo y julio del 2015, con el fin de obtener la prevalencia nacional de Campylobacter spp. en pollo de engorde para consumo humano. Se utilizó un diseño de muestreo simultáneo en tres niveles de la cadena productiva avícola costarricense: 152 muestras en granja (CC), 104 en planta (CA) y 96 en puntos de venta (PV). Se elaboró formularios de variables asociadas al manejo en las plantas (CC y CA) y los PV. Los formularios, en las plantas, incluyeron variables relacionadas con datos generales como: ubicación y volumen de producción, buenas prácticas higiénicas y de manufactura (BPHM), procesos, y HACCP. En PV se evaluó aspectos relacionados con BPHM, temperatura del producto, y precio por $\mathrm{kg}$ de pollo limpio, entre otros. Se determinó como factores de riesgo los siguientes: tiempo de espera de animales vivos en andén $>60$ minutos ( $R P=2.28$, IC 95\% 1.23-4.21); velocidad de proceso $<120$ aves $/ \mathrm{min}(R P=1.51$, IC95\% 1.09-2.10); temperatura del agua del chiller $\leq 0.5^{\circ} \mathrm{C}$ (RP 1.60, IC95\% 1.18-2.17); sin separación física entre matrices de diferente origen animal en $P V(R P=1.78, I C 95 \% 1.43-2.20)$ y precio en PV $\leq 2000$ colones/kg de pollo limpio ( $R P=1.73$, IC95\% 1.21-2.46). La prevalencia encontrada de Campylobacter spp. es alta. Representa un peligro para la salud pública en Costa Rica. Por tal motivo, se debe implementar intervenciones bactericidas más eficientes contra este agente y mejorar las BPHM en cada eslabón de la cadena de producción.

Palabras clave: Campylobacter spp., factores de riesgo, avicultura, puntos de venta, inocuidad.

Abstract. Campylobacter spp. are bacteria known to cause foodborne diseases in both developed and developing countries. A cross-sectional study was conducted between March and July 2015 to determine the national prevalence of Campylobacter spp. in broilers for human consumption. A simultaneous sampling design was used in three levels of the Costa Rican poultry production 
chain: 152 farm samples (CC), 104 plant samples (CA) and 96 retail store samples (PV). For this research, questionnaires were prepared with variables related to plant (CC and CA) and PV management. The plant questionnaire included variables related to general data such as location and volume of production, good hygiene and manufacturing practices (GHMP), as well as processes and HACCP. In PV aspects such as the following were evaluated: GHMP, product temperature, and price per $\mathrm{kg} / \mathrm{chicken}$. The following were identified as risk factors: resting period of live animals in platform $>60$ minutes $(P R=2.28,95 \% \mathrm{Cl} 1.23-4.21)$, processing speed $<120 \mathrm{birds} / \mathrm{min}(\mathrm{PR}=1.51,95 \% \mathrm{Cl} 1.09-2.10)$, chiller water temperature $\leq 0.5^{\circ} \mathrm{C}(\mathrm{PR}$ 1.60, 95\% Cl 1.18-2.17), no physical separation between different animal matrices in PV (PR $=1.78,95 \% \mathrm{Cl} 1.43-2.20)$ and price in $\mathrm{PV} \leq 2000$ colones $/ \mathrm{kg}$ chicken $(\mathrm{PR}=1.73,95 \% \mathrm{Cl} 1.21-$ 2.46). The prevalence of Campylobacter spp. in the Costa Rican poultry production chain is high and represents a risk to public health. For this reason, more efficient bactericidal interventions against Campylobacter spp. should be implemented, as well as improvements of the GHMP in every stage of the production chain.

Keywords: Campylobacter spp., risk factors, poultry production, retail store, food safety.

\section{Introducción}

Campylobacter spp. es una bacteria que se ha destacado por su alta ocurrencia como causa de infecciones transmitidas por alimentos (ETA), tanto en países desarrollados como en vías de desarrollo (CDC 2014; EFSA 2015; Macé et al. 2015). Se estima que puede llegar a afectar por encima de 2.4 millones de personas por año en EEUU (CDC 2014). La mayor parte de las ETA, causadas por este agente, son transmitidas por carne de aves contaminadas (Fischer et al. 2013); aunque se ha determinado también en la leche cruda y agua contaminada como fuentes de infección (Indikova et al. 2015).

Costa Rica presenta un consumo per cápita anual de $23.42 \mathrm{~kg}$ de productos y subproductos de la cadena de carne avícola (Wright 2010). Un alto consumo de carne de ave, sin el debido tratamiento en la producción, venta o preparación para consumo, implica un mayor riesgo de infección.

En Costa Rica, no se ha realizado estudios que determinen los factores de riesgo para la ocurrencia de Campylobacter spp. en granjas, plantas de proceso, ni en puntos de venta. Sin embargo, en otros países se ha demostrado que las carcasas de lotes, con contenido cecal (CC) positivo a Campylobacterspp., tienen mayor cantidad de UFC (unidades formadoras de colonias) por gramo, que aquellas con CC negativo (Rosenquist et al. 2006, Allen et al. 2007, Reich et al. 2008, Hue et al. 2011). Además, se ha descrito que lotes, los cuales no se sacrifican de primero en el horario diario, tienen mayor riesgo de resultar positivos a Campylobacter spp. (Hue et al. 2010). Otros factores de riesgo asociados son: mantener una temperatura mayor a $15^{\circ} \mathrm{C}$ en la sala de eviscerado (Hue et al. 2010); presentar el yeyuno lleno al momento de eviscerado (Arsenault et al. 2007) y contaminación visible después de la evisceración (Hue et al. 2010).

El objetivo de este estudio consiste en determinar los factores asociados a la contaminación con Campylobacter spp. termotolerante en la cadena de producción de pollo para consumo humano en Costa Rica, tomando como trazadores la granja, la planta de proceso y el punto de venta, en forma simultánea. 


\section{Materiales y Métodos}

\section{Tipo de estudio y lugar de muestreo}

Se realizó un estudio transversal, entre marzo y julio del 2015, para obtener la prevalencia nacional de $C$. jejuni y $C$. coli en pollo para consumo humano, utilizando un diseño de muestreo en tres niveles de la cadena productiva avícola costarricense: las granjas, las plantas procesadoras y los puntos de venta. Se eligió este tipo de estudio debido a que permite estimar la magnitud y distribución del agente patógeno, caracterizando el estado de sanidad del pollo. Se definió como caso toda ave de producción que haya sido muestreada dentro de la investigación durante el período transcurrido entre marzo y julio del 2015, en las plantas y puntos de venta del territorio nacional, que resulte positiva para Campylobacter spp (Zumbado 2016).

Con base en las ocurrencias encontradas por Zumbado et al. (2014), se calculó un tamaño de muestra para obtener una prevalencia nacional en cada eslabón del proceso por estudiar. Se utiliza el programa WinEpiscope® basado en la fórmula para determinar prevalencia, con una población aproximada de aves procesadas en un año de 60938114 (Wright 2010); una prevalencia esperada de $80 \%$ para lotes de aves por medio del contenido cecal; $40 \%$ para carcasa y $50 \%$ para puntos de venta, asumiendo un error aceptado de $10 \%$ y un nivel de confianza de $95 \%$. Se determinó que el tamaño de muestra requerido fuera de 63 muestras de lotes de aves (126 de contenido cecal), 99 muestras de enjuagues de carcasa y 96 muestras de puntos de venta, para un total de 321. El número de muestra de contenido cecal es el doble para determinar la presencia del agente en los lotes de aves, esto debido a que, en cada lote, se tomó dos muestras, para evitar la pérdida de material por contaminación durante el análisis (Zumbado 2016).

\section{Granjas}

Se analizó aves de engorde destinadas para consumo humano, con edad entre 35 y 40 días, con un peso vivo entre 1500 a 3000 gramos. El grueso de la producción avícola, en Costa Rica, está concentrado entre pocos productores de mayor escala, los cuales manejan estrictas normas de bioseguridad y protocolos de manejo y nutrición para el engorde de las aves; sin embargo, no existe uniformidad entre las granjas de cría a nivel nacional. Se consideró que la prevalencia de este patógeno no variará durante los períodos de producción o temporadas climáticas del año, debido al tipo de sistema de producción de pollo a nivel nacional, mayoritariamente intensivo y las características propias de Campylobacter spp. (Zumbado 2016).

\section{Plantas de proceso}

La distribución dela cantidad de muestras en plantas de proceso se realizó proporcionalmente al volumen de producción; además, se clasificó las plantas de proceso según su volumen de producción: volumen bajo (BV) (menor o igual a 4000 aves procesadas por semana), 
volumen mediano (MV) (entre 4001 a 100 000) y volumen alto (AV) (mayor a 100 000) (Zumbado 2016).

Las plantas clasificadas como alto volumen (AV) tienen en común contar con programas de buenas prácticas de higiene y manufactura (BPHM); un sistema de análisis de peligros y puntos críticos de control (HACCP, por sus siglas en inglés). Además, con la inspección veterinaria oficializada. Tres plantas utilizan un enjuague con ácido peracético posterior al chiller. Las plantas de mediano volumen (MV) y bajo volumen (BV) cuentan con BPHM e inspección veterinaria privada; uno de los establecimientos de MV (planta E) utiliza un enjuague con ácido peracético, previo a la entrada al chiller. Todas las plantas de proceso utilizan cloro en el agua del chiller entre 20 y 50 ppm (Zumbado 2016).

\section{Puntos de Venta}

La distribución de las muestras por PV se realizó proporcionalmente a la población de cada provincia, tomando como referencia tres cantones, a su vez, los tres distritos más poblados de cada uno (INEC 2011). De esta manera, se aseguró muestrear las zonas en las cuales se comercializa la mayor cantidad de producto; por ende, mayor cantidad de personas podría estar expuestas al Campylobacter (Zumbado 2016).

Los puntos de venta se clasificaron como Fuera de Mercado (FM) y Dentro de Mercado (DM). Los establecimientos FM tienen una única entrada, están localizados frente a una calle transitada por vehículos automotores y personas; además, tienen un suministro de agua separado de otros negocios; mientras que los DM, están ubicados dentro de un mercado municipal, los cuales tienden a ser cerrados y permiten únicamente el tránsito de personas. Además, comparten el suministro de agua y servicios sanitarios con otros establecimientos del mercado (Zumbado 2016).

\section{Recolección de las muestras}

Se recolectó en total 352 muestras, las cuales se distribuyeron de acuerdo con las frecuencias esperadas (Zumbado et al. 2014) ( $n=152$ muestras de granja, $n=104$ muestras de planta de proceso y $n=96$ muestras de punto de venta). Se recolectó tres tipos de muestras: ciegos para extraer el contenido cecal (CC) (muestra representativa de la granja), enjuague de la carcasa a la salida del sistema de enfriamiento (CA) (muestra representativa de la planta de proceso), y enjuague de la carcasa en el punto de venta (PV).

\section{Contenido cecal}

Las aves procedentes de cada granja fueron identificadas en planta, de manera que, tanto las muestras de CC como la de CA, pertenecieran a la misma granja. Se recolectó el ciego de dos aves inmediatamente después de la evisceración; cada ciego se depositó en una bolsa plástica estéril, debidamente identificada. 
Cuando una muestra de contenido cecal resultó positiva, se infirió que el lote, del cual proviene, fue positivo, debido a la metodología de producción de aves: "Todo dentro, todo fuera" y las características de la infección por Campylobacter spp. en las aves. "Todo dentro, todo fuera" se refiere a que las aves de un mismo lote reciben el mismo manejo en términos de vacunas, alimentación y medicamentos, entre otros; por lo cual es muy probable que de estar el microorganismo circulando en un ave, éste se encuentre en el resto de aves (Zumbado 2016).

\section{Enjuague de carcasa}

Al finalizar el período que transcurre el ave en el sistema de enfriamiento (chiller), de la línea de producción, se realizó el muestreo de la carcasa por medio del método de enjuague (USDA 2008). Para cada carcasa muestreada se midió la temperatura interna en la profundidad de la pechuga, por medio de un termómetro apto para alimentos calibrado y desinfectado.

\section{Enjuague de carcasa adquirida en puntos de venta}

En el PV, la muestra que corresponde a una carcasa (conocida como pollo limpio), se recolectó en la manera usual de compra, la cual se transportó en la bolsa suministrada por el comercio. A la muestra se midió la temperatura inmediatamente después de recibida, en la profundidad de la pechuga mediante un termómetro apto para alimentos, calibrado y desinfectado. Una vez en el laboratorio, cada carcasa comprada recibió un enjuague como el descrito (Zumbado 2016).

Todas las muestras recolectadas se transportaron al laboratorio de Bacteriología de la Escuela de Medicina Veterinaria (EMV) de la Universidad Nacional (UNA), a una temperatura no mayor a $4^{\circ} \mathrm{C}$, para su análisis.

\section{Recolección de la información de las variables por estudiar}

Se elaboró formularios de variables asociadas al manejo en las plantas y a los PV. Estos fueron llenados a partir de las observaciones del investigador e información suministrada por colaboradores de las plantas de cosecha y por los dependientes en los PV.

La variable dependiente fue la presencia de colonias Campylobacter spp. en las muestras recolectadas a partir de los tres puntos de muestreo. Esta es una variable de tipo cualitativa, con escala nominal. Los formularios, en las plantas, incluyeron variables relacionadas con datos generales como ubicación y volumen de producción; características propias de los procedimientos de buenas prácticas de manufactura, establecidos según la normativa nacional (SENASA 2013) y códigos internacionales (Códex Alimentario 2005). Entre los aspectos analizados están: ubicación de las granjas proveedoras, tiempo de espera de las aves antes de sacrificio, si la evisceración es automática, si se observa ruptura de intestino después de la evisceración, velocidad de proceso, concentración de cloro en el agua del chiller, temperatura del agua del chiller, temperatura de la carcasa, uso y concentración de ácido peracético.

Se visitó 96 establecimientos de venta en las siete provincias de Costa Rica. En cada expendio se evaluó aspectos relacionados con las buenas prácticas de manufactura (BPM), 
entre estas: uso de cubrebocas, uso de cofia, uso de guantes desechables, lavado de manos, tipo de jabón utilizado (en barra o líquido), manipulación de dinero por parte del dependiente, tipo de productos comercializados, separación física con carnes de diferente origen animal, limpieza de la cámara de frío y limpieza general del establecimiento; además, características propias del producto como: la temperatura (medida en la profundidad de la pechuga), precio por kg de pollo limpio y riesgo de contaminación cruzada.

Para la variable, separación física de carnes de diferente origen animal, se clasificó el riesgo como: cámara de frío solo para pollo (sin riesgo), separación física de otras carnes dentro de la misma cámara, por ejemplo: el uso de bandejas diferentes (grado medio de riesgo) y sin separación física entre carnes de distinto origen (grado alto de riesgo).

\section{Cultivo de Campylobacter spp.}

Para el procesamiento de los enjuagues de carcasa de pollo se siguió el protocolo ISO 10272-1:2006 modificado por el Departamento Agrícola de Estados Unidos (USDA, por sus siglas en inglés) (USDA, 2008). Para la detección de Campylobacter sp. a partir de los ciegos, el CC fue homogenizado en $20 \mathrm{ml}$ de BPW; $1 \mathrm{ml}$ de la mezcla se trasfirió a $9 \mathrm{ml}$ de caldo Preston suplementado. Los periodos de incubación, tiempos y pruebas bioquímicas de tamizaje fueron las mismas empleadas para los enjuagues de carcasa de pollo (Zumbado et al. 2014; Zumbado 2016).

\section{Análisis estadístico}

Se calculó: medidas de frecuencias relativas y absolutas, las variables cualitativas nominales y ordinales, así como cuantitativas discretas. Se obtuvo las medidas de tendencia central y de dispersión para las variables cuantitativas continuas y discretas.

Para identificar los factores de asociación con la presencia de Campylobacter spp. en las aves muestreadas, se calculó medidas de asociación como Chi cuadrado de Pearson y la razón de prevalencias utilizando tablas de contingencia (2xk) en un esquema univariado, utilizando los software InfoStat ${ }^{\circledR}$ (InfoStat, FCA-UNC, Córdoba, Argentina) y WinEpiscope 2.0 ® (EPIDECON, Zaragoza, España). Se estableció como valor umbral de significancia ( ) el 5\%; así como la no inclusión del valor 1 en el intervalo de confianza al $95 \%$. Al mismo tiempo, se realizó un análisis multivariado uitilzando el software Egret@ 2.0.31 (Cytel Software Corporation, EEUU).

\section{Resultados}

\section{Prevalencia de Campylobacter spp.}

Se obtuvo una prevalencia general para Campylobacter spp. de $59.37 \%$ (209/352, IC 95\% 54.24\%-64.51\%). Se determinó una prevalencia de 57.24\% (87/152, IC 95\% 49.37\%$65.10 \%$ ) en CC, $61.53 \%$ (64/104, IC 95\% 52.19\%-70.89\%) en CA y $60.42 \%$ (58/96, IC $95 \%$ 50.63\%-70.20\%) en PV (Zumbado 2016). 


\section{Factores asociados a contenido cecal}

Se determinó que el tiempo de espera promedio de las aves, previo al sacrificio, en el andén, fue de 96.65 minutos (DE 104.81, mín 10 - máx 450.5 minutos). Se determinó el tiempo de espera > a 60 minutos como factor de riesgo con una RP 2.28 (Cl 95\% 1.23-4.21).

\section{Factores asociados a enjuague de carcasa}

Durante el proceso de evisceración, se observó ruptura de intestinos de las aves en un $39.42 \%$ ( $n=41 / 104) ;$ sin embargo, este aspecto no se asoció estadísticamente con el resultado positivo.

Las variables continuas fueron analizadas mediante estadística descriptiva (Cuadro 1); las variables reglamentadas, como temperatura de la carcasa a la salida del chiller $\left(\leq 4.4^{\circ} \mathrm{C}\right)$ y la concentración del cloro en el agua de la carcasa (20-50 ppm), se encontró según el reglamento (SENASA 2013); excepto en una visita en la cual la concentración de cloro estuvo en $0.00 \mathrm{ppm}$.

Cuadro 1. Medidas resumen de variables numéricas asociadas a la muestra de enjuague de carcasa, en las plantas de proceso avícola, durante el período de marzo a julio del 2015, en Costa Rica.

\begin{tabular}{lcccc}
\hline \multicolumn{1}{c}{ Variable } & Media & DE & Mín & Máx \\
\hline Temperatura del agua en chiller $\left({ }^{\circ} \mathrm{C}\right)$ & 0.84 & 0.51 & 0.00 & 2.50 \\
Temperatura CA $\left({ }^{\circ} \mathrm{C}\right)$ & 2.80 & 0.81 & 1.20 & 4.40 \\
Concentración de Cloro $(\mathrm{ppm})$ & 34.56 & 13.22 & 0.00 & 50.00 \\
Concentración Ácido Peracético (ppm) & 131.47 & 55.56 & 0.00 & 240.00 \\
Velocidad de Proceso (pollos/minuto) & 121.02 & 34.60 & 25.00 & 150.00 \\
\hline
\end{tabular}

No se halló valores cercanos a la significancia para ruptura de intestino, temperatura de CA, concentración de cloro, concentración de ácido peracético; pero sí se encontró asociación estadística en el caso de velocidad de proceso $<120$ aves/min, así como para temperatura del agua del chiller $\leq 0.5^{\circ} \mathrm{C}$ (Cuadro 2).

\section{Factores asociados con enjuague de carcasa obtenida en punto de venta}

Ninguno de los aspectos relacionados con las BPM fue asociado con la ocurrencia de Campylobacter spp., excepto por la separación física entre el pollo y productos de distinto origen animal comercializado a granel, como res o cerdo. No se determinó una asociación estadística para la variable en general; sin embargo, los valores son muy cercanos a la significancia (Cuadro 2). Al estratificar esta variable, se encontró un valor de RP significativo para la observación sin separación física (Cuadro 2). 
De manera semejante, en el caso de la evaluación de lasvariables referidas a las características propias del producto, no se reflejó asociaciones estadísticas; excepto para la variable precio. Los precios por kg de pollo (pollo limpio) variaron entre los establecimientos, con un promedio de 2166.47 colones (DE 391.30, mínimo 1160-máximo 3148). Se determinó un RP de 1.72, para precios $\leq 2000$ colones por kg de pollo limpio (Cuadro 2).

La temperatura de la carcasa, inmediatamente posterior a su compra, fue en promedio $6.00^{\circ} \mathrm{C}$ (DE 3.31, mínimo -1.11 máximo 15.55); se excluyó de esta variable 4, carcasas que se encontraban congeladas al momento de la compra, 3 de ellas resultaron negativas a la presencia de Campylobacter spp. No se determinó asociación significativa entre la temperatura de la carcasa y el resultado encontrado.

Cuadro 2. Valores de Chi cuadrado de Pearson y RP (IC 95\%) para algunas variables evaluadas en planta y puntos de venta, durante marzo a julio del 2015, con respecto al resultado positivo de Campylobacter spp.

\begin{tabular}{lccc}
\hline Variable & N positivas & $\begin{array}{c}\text { Valor Chi Pear- } \\
\text { son }(\mathrm{p})\end{array}$ & $\begin{array}{c}\text { Razón de Prevalencias } \\
(\mathrm{IC} 95 \%)\end{array}$ \\
\hline Ruptura de Intestino & 26 & $0.10(0.751)$ & $1.051(0.77-1.43)$ \\
Velocidad de Proceso $<120$ aves/min & 22 & $6.27(0.0123)$ & $1.51(1.09-2.10)$ \\
Temperatura del agua del chiller $\leq 0.5^{\circ} \mathrm{C}$ & 28 & $9.25(0.0024)$ & $1.60(1.18-2.17)$ \\
Separación física con carnes de distinto & 22 & $5.75(0.0564)$ & $1.2222(0.92-1.62)$ \\
origen & & & \\
Sin separación física & 8 & & $1.7778(1.43-2.21)$ \\
Precio $\leq 2000$ colones/kg de pollo limpio & 37 & $9.21(0.0024)$ & $1.7267(1.21-2.46)$ \\
\hline
\end{tabular}

Con respecto a la contaminación cruzada, aunque no se determinó asociación estadística, sí se observó conductas reconocidas como de peligro en algunos de los PV visitados, entre éstas: producto empacado en el piso, uso de tarimas de madera, manipulación de matrices de distinto origen sin lavado de manos previo o intercambio de guantes, presencia de moscas dentro de las cámaras de frío, cámaras de frío con el vidrio quebrado, uso de la tabla de picar sin lavar entre usos y canal de cerdo colgada en área abierta al público.

No hubo resultados estadísticamente significativos al realizar el análisis multivariado.

\section{Discusión}

La asociación estadística encontrada en este estudio, con respecto al tiempo de espera en el andén previo al sacrifico, mayor a 60 minutos (RP 2.28), se relaciona con otras investigaciones, las cuales han encontrado que los recuentos de Campylobacter spp. aumentan posterior al transporte, en comparación con recuentos realizados en granja. A su vez, éstos estudios demostraron que el tiempo de reposo en el matadero no significó una reducción en los recuentos (Whyte et al. 2001, Wesley et al. 2005). Paralelamente, se ha determinado que el estrés inducido por el transporte aumenta la proporción de Campylobacter spp. presente 
en el ave, que puede, consecuentemente, resultar en mayor contaminación de la carcasa (Whyte et al. 2001).

Por otra parte, se ha observado que la prevalencia de Campylobacter spp. tiende a decrecer con mayor número de aves por galpón, mientras que la prevalencia de Salmonella aumenta mientras mayor sea la cantidad de aves en cada galpón (Franz et al. 2012); sin embargo, este aspecto no fue evaluado en este estudio.

La ruptura de intestinos de las aves, observada durante el proceso de evisceración (39.42\%), no se asoció estadísticamente con el resultado positivo. Sin embargo, con respecto a la contaminación causada por una inadecuada evisceración, se ha determinado que las carcasas de lotes con CC positivo a Campylobacter spp., tienen mayor cantidad de UFC por gramo, que aquellas con CC negativo (Rosenquist et al. 2006, Allen et al. 2007, Reich et al. 2008, Hue et al. 2011). Esto demuestra que la evisceración, en las plantas de cosecha, es el paso en la cadena de proceso que más contribuye a diseminar la contaminación (Hue et al. 2010, Hue et al. 2011). Asimismo, se ha descrito que los lotes que no se sacrifican de primero, en el horario cotidiano, tienen mayor riesgo de resultar positivos a Campylobacter spp. $(\mathrm{OR}=3.5, \mathrm{p}<0.05)$. Otros factores de riesgo, asociados, son mantener una temperatura mayor a $15^{\circ} \mathrm{C}$ en la sala de eviscerado $(\mathrm{OR}=3.1, \mathrm{p}<0.05)$ y contaminación fecal visible después de la evisceración (OR=2.6, $\mathrm{p}<0.05$ ) (Hue et al. 2010).

Los resultados de esta investigación no demuestran diferencias significativas con respecto al uso y concentraciones de cloro y/o ácido peracético, y el resultado positivo; estos resultados son similares a los encontrados por otro estudio, el cual determinó que el nivel de cloro y la temperatura del agua no tuvieron efecto sobre el recuento total aerobio (RTA), Escherichia coli o Campylobacter spp. recuperados después del proceso de enfriamiento (Northcutt et al. 2005). Sin embargo, otras investigaciones han demostrado un efecto de reducción de los recuentos de Campylobacter spp. Y E. coli, con el uso de clorito de sodio acidificado, utilizado posterior al chiller, ya sea mediante inmersión o rociado (Oyarzaval et al. 2004). Se ha comprobado, también, que el agua electrolizada (AE) tiene mayor efecto bacteriostático que la aplicación de cloro por sí solo $(25 \mathrm{mg} / \mathrm{l}$ de cloro residual) in vitro (Park et al. 2002).

El ácido peracético ha probado ser efectivo en concentraciones bajas $(0.0025 \%)$ para disminuir los niveles de Salmonella spp.; mientras que para reducir Campylobacter spp. es necesario emplear concentraciones de $0.02 \%$. Adicionalmente, se determinó que el uso de ácido peracético, en concentraciones $\geq 0.015 \%$, puede extender la vida de anaquel de los productos (Bauermeister et al. 2008). De igual manera, el uso de rociado con cloruro cetilpiridina post chiller, también demostró una reducción significativa de Campylobacter spp. ySalmonella spp. (Wideman et al. 2015).

En este estudio no se determinó asociación estadística con un nivel de confianza del 95\% entre la temperatura de la carcasa y la presencia de Campylobacter spp., pero sí para la temperatura del agua del chiller. En este caso, se observó un mayor riesgo cuando la temperatura del agua es $\leq 0.5^{\circ} \mathrm{C}$ (RR 1.60). Sin embargo, estudios in vitro han demostrado 
que se reduce significativamente el recuento de Campylobacterspp., manteniéndolo a $\leq 4^{\circ} \mathrm{C}$ por un período de $48 \mathrm{hrs}$. (Sollow et al. 2003). Adicionalmente, se ha mencionado que los sistemas de enfriamiento, mediante aire, resultan en menor incidencia de Salmonella spp. y Campylobacterspp. que los sistemas de enfriamiento por inmersión en agua, lo cual sugiere que la contaminación cruzada puede ser más prevalente para las carcasas enfriadas por inmersión (Sánchez et al. 2002). El tiempo transcurrido, en el uso del agua del chiller, se ha asociado a menor disminución en los recuentos de Campylobacter spp., sobre todo por la inactivación del efecto bactericida del cloro (Yang et al. 2001; Bucher et al. 2015).

Por otra parte, estudios han sugerido que el recuento de Campylobacter spp. disminuye al utilizar temperaturas del agua de escaldado entre 50 y $60^{\circ} \mathrm{C}$ (Yang et al. 2001, Lehner et al. 2014), pero este aspecto no fue evaluado en este estudio.

Con respecto a los resultados en PV, no se observó una asociación estadística a un nivel de confianza del $95 \%$ entre la temperatura de la carcasa y el resultado. Sin embargo, sí se determinó, en un estudio realizado en Chile, que la congelación tiene un efecto bacteriostático/bactericida sobre el Campylobacter spp., por lo cual, reduce el nivel de contaminación. En consecuencia, se estima que los pollos congelados presentan un riesgo menor a aquellos que se venden y almacenan refrigerados (Figueroa 2006). Aspecto observado en los pollos congelados que se analizaron en este estudio.

Adicionalmente, se ha sugerido una correlación positiva entre la presencia de Campylobacter spp. en pacientes infectados y el consumo de carne de pollo fresco (Lindmark et al. 2009). Por otra parte, en Nueva Zelanda, los casos de Campylobacter spp. en humanos, asociados al consumo de carne de aves, se describe mayormente en áreas urbanas, en comparación con las rurales; particularmente los niños en áreas rurales demostraron tener mayor riesgo de infectarse con cepas provenientes de rumiantes (Mullner et al.2010).

Además, se ha asociado la presencia de Campylobacter spp. a la carne de aves, canales de cerdo y de res (Oporto \& Hurtado 2011, Abley et al. 2012, Mattheus et al. 2012), por lo cual, la contaminación cruzada, al existir contacto directo con carne de diferentes orígenes en el punto de venta, es altamente probable; factor que se demostró en este estudio cuando no había separación física entre las carnes (RP 1.77).

La prevalencia de Campylobacterspp., demostrada en esta investigación, es alta. Representa un riesgo para la salud pública en Costa Rica. Las infecciones por Campylobacterspp. generan un problema serio para la salud humana y la economía a nivel mundial. El papel clave de las aves de corral, como un reservorio de la infección, está claramente establecido. Existe la necesidad apremiante de vacunas o tratamientos para limitar la entrada de Campylobacter spp. en la cadena alimentaria y el ambiente (Buckley et al. 2010).

Las medidas de control, que han demostrado ser efectivas para reducir la contaminación con Salmonellaspp., no han tenido el mismo efecto reductor en Campylobacterspp.; por tal motivo, se necesita evaluar nuevas intervenciones que se complementen o reduzcan los niveles de ésta bacteria (Wilson 2002). Por lo demás, ninguna medida ha demostrado ser suficiente por 
sí sola, para reducir Campylobacter spp. en la pre-cosecha, cosecha, o post-cosecha; pero una combinación de medidas, en todos los niveles de producción, podría ser beneficiosa.

Además, se observó que las prácticas de manipulación de alimentos, en los puntos de venta visitados, no son los adecuados, de acuerdo con lo recomendado por el Codex Alimentarius. Es necesaria una mayor rigurosidad en la inspección y auditorias por parte de la entidad competente. Aunque no se haya demostrado asociación estadística entre la mayoría de estas prácticas y la presencia de Campylobacter spp., no se descarta que ciertas conductas observadas sean de riesgo para este y otros agentes causantes de ETA.

\section{Agradecimientos}

El financiamiento para este estudio fue otorgado por la Universidad Nacional, mediante concurso FIDA(FondolnstitucionalparaelDesarrolloAcadémico).Seagradeceprofundamente al equipo de trabajo del Laboratorio de Bacteriología de la Escuela de Medicina Veterinaria de la Universidad Nacional, al equipo de trabajo de SENASA y a las estudiantes Mónica Jiménez Mora y Jessica Abarca Gómez. Además, se agradece a los médicos veterinarios y al personal de los establecimientos procesadores por su colaboración.

\section{Referencias}

Abley, M., Wittum, T., Funk, J. \& Gebreyes, W. 2012. Antimicrobial susceptibility, pulsedfield electrophoresis, and multi-locus sequence typing of Campylobacter coli in swine before, during, and after the slaughter process. Foodborne Pathogens and Disease. 9(6): 506- 512.doi:10.1089/fpd.2011.1053

Allen, V. M., Bull, S.A., Corry, J.E.L., Domingue, G., Jorgensen, F., Frost, J.A., Whyte, R., Gonzalez, A., Elviss, N. \& Humphrey, T.J. 2007. Campylobacter spp. contamination of chicken carcasses during processing in relation to flock colonisation. Int. Journal of Food Microbiol.113 (1): 54-61. doi:10.1016/j.ijfoodmicro.2006.07.011

Arsenault, J., Letellier, A., Quessy, S. \& Boulianne, M. 2007. Prevalence and risk factors for Salmonella and Campylobacter spp. carcass contamination in broiler chickens slaughtered in Quebec, Canada. Journal of Food Protection 8:1784-1993.

Bauermeister, L. J., Bowers, J. W. J., Townsend, J. C. \& McKee, S. R. 2008. The microbial and quality properties of poultry carcasses treated with peracetic acid as an antimicrobial treatment. Poultry Science, 87(11): 2390-2398. http://doi.org/10.3382/ps.2008-00087

Bucher, O., Waddell, L., Greig, J. \& Smith, B. A. 2015. Systematic review-meta-analysis of the effect of chilling on Campylobacter spp. during primary processing of broilers. Food Control, 56: 211-217. http://doi.org/10.1016/j.foodcont.2015.03.032

Buckley, A.M., Wang, J., Hudson, D.L., Grant, A.J., Jones, M.A., Maskell, D.J. \& Stevens M.P. 2010. Evaluation of live-attenuated Salmonella vaccines expressing Campylobacter antigens for control of C.jejuniin poultry. Vaccine 28(4): 1094-1105. doi.org/10.1016/j.vaccine.2009.10.018 
Centers for Disease Control and Prevention. 2014. Campylobacter: General information. Disponible en http://www.cdc.gov/nczved/divisions/dfbmd/diseases/campylobacter. Revisado 19 de enero 2016.

Codex Alimentario. 2005. CAC/RCP 58/2005: Código de Prácticas de Higiene para la Carne. Disponible en: http://www.codexalimentarius.org/normas-oficiales/lista-de-las-normas.

European Food Safety Authority y European Centre for Disease Prevention and Control (EFSA). 2015. The European Union summary report on trends and sources of zoonoses, zoonotic agents and food-borne outbreaks in 2014. EFSA Journal 13 (12): 4329. doi:10.2903/j.efsa.2015.4329

Figueroa A. 2006. Presencia de Campylobacter jejuni en carne de ave congelada en una planta procesadora de la región metropolitana. (Tesis de Licenciatura). Universidad de Chile. Santiago.

Fischer, S., Kittler, S., Klein, G. \& Glünder, G. 2013. Microplate-test for the rapid determination of bacteriophage-susceptibility of Campylobacter isolates-development and validation. PLOS ONE, 8(1).

http://doi.org/10.1371/journal.pone.0053899

Food and Agricultural Organization (FAO) \& World Health Organization (WHO). 2009. Microbiological risk assessment series 19: Salmonella and Campylobacter in chicken meat. Disponible en: www.who.int/foodsafety/publications/micro/MRA11_En.pdf. Revisado 24 enero 2016.

Franz E., van der Fels-Klerx, H.J., Thissen, J. \& van Asselt, E.D. 2012. Farm and slaughterhouse characteristics affecting the occurrence of Salmonella and Campylobacter in the broiler supply chain. Poultry Science 91: 2376-2381.doi:10.3382/ps.2009-00367

Hue, O., Le Bouquin, S., Laisney, M.J., Allain, V., Lalande, F., Petetin, I., Rouxel, S., Quesne, S., Gloaquen P.Y., Picherot, M., Santolini, J., Salvat, G., Bougeard, S. \& Chemaly, M. 2010. Prevalence of and risk factors for Campylobacter spp. contamination of broiler chicken carcasses at the slaughterhouse. Food Microbiol 27 (8): 992-9. doi: 10.1016/j. fm.2010.06.004

Hue, O., Allain, V., Laisney M.J., Le Bouquin, S., Lalande, F., Petetin, I., Rouxel, S., Quesne, S., Gloaquen, P.Y., Picherot, M., Santolini, J., Bougeard, S., Salvat, G. \& Chemaly M. 2011. Campylobacter contamination of broiler caeca and carcasses at the slaughterhouse and correlation with Salmonella contamination. Food Microbiol. 28 (5): 862-8. doi: 10.1016/j.fm.2010.11.003

Indikova, I., Humphrey, T. J. \& Hilbert, F. 2015. Survival with a helping hand: Campylobacter and microbiota. Frontiers in Microbiology, 6(NOV). http://doi.org/10.3389/ fmicb.2015.01266

Instituto Nacional de Estadística y Censos. 2011. Cuadro 1: Costa Rica, Indicadores demográficos según cantón (Censo2011). Consultado: 21 de junio del 2014., http:// www.inec.go.cr/Web/Home/GeneradorPagina.aspx 
Lehner, Y., Reich, F. \& Klein, G. 2014. Influence of process parameter on Campylobacter spp. counts on poultry meat in a slaughterhouse environment. Curr. Microbiol. 69(3): 240244. doi 10.1007/s00284-014-0575-y

Lindmark, H.,Boqvist, S., Ljungström, M.,Ågren, P., Björkholm, B.\&Engstrand, L. 2009. Risk Factors for Campylobacteriosis: an Epidemiological Surveillance Study of Patients and Retail Poultry. J. Clin. Microbiol. 47(8): 2616-2619. doi:10.1128/JCM.00826-09

Macé, S., Haddad, N., Zagorec, M. \& Tresse, O. 2016. Influence of measurement and control of microaerobic gaseous atmospheres in methods for Campylobacter growth studies. Food Microbiology, 52: 169-176. http://doi.org/10.1016/j.fm.2015.07.014

Mattheus, W., Botteldoorn, N., Heylen, K., Pochet, B. \&Dierick, K. 2012. Trend Analysis of antimicrobial resistance in Campylobacter jejuni and Campylobacter coli isolated from Belgian pork and poultry meat products using surveillance data of 2004-2009. Foodbourne Pathogens and Disease. 9(5): 465-472.doi:10.1089/fpd.2011.1042

Mullner, P., Shadbolt, T., Collins-Emerson, J.M., Midwinter, A.C., Spencer, S.E.F., Marshall, J., Carter, P.E., Campbell, D.M., Wilson, D.J., Hathaway, S., Pirie, R. \& French, N.P. 2010. Molecular and spatial epidemiology of human campylobacteriosis: source association and genotype-related risk factors. Epidemiology and Infection 138(10): 1372-1383. doi.org/10.1017/S0950268809991579

Northcutt, J.K., Smith, D.P., Musgrove, M.T., Ingram, K.D. \& Hinton, A. 2005. Microbiological impact of spray washing broiler carcasses using different chlorine concentrations and water temperatures. Poultry Science 84: 1648-1652.doi:10.1093/ps/84.10.1648

Oporto, B. \& Hurtado, A. 2011. Emerging thermotolerant Campylobacter species in healthy ruminants and swine. Foodborne Pathogens and Disease 8(7): 807-813.doi:10.1089/ fpd.2010.0803.

Oyarzabal, O.A., Hawk, C., Bilgili, S., Warf, C.C., Kemp \& G.K. 2004. Effects of postchill application of acidified sodium chlorite to control Campylobacter spp. and Escherichia coli on commercial broiler carcasses. Journal of Food Protection 10: 2092-2353.

Park, H., Hung, Y. \& Brackett. R.E. 2002. Antimicrobial effect of electrolyzed water for inactivating Campylobacter jejuni during poultry washing. Int. Journal of Food Microbiol. 72(1-2): 77-83.

Reich, F., Atanassova, V., Haunhorst E. \& Klein, G. 2008. The effects of Campylobacter numbers in caeca en the contamination of broiler carcasses with Campylobacter. International Journal of Food Microbiology 127 (1-2):116-120. doi:10.1016/j. ijfoodmicro.2008.06.018.

Rosenquist, H., Sommer, H.M., Nielsen, N.L. \& Christensen, B.B. 2006. The effect of slaugher operations on the contamination of chicken carcasees with thermotolerantCampylobacter. Int. Journal of Food Microbiol. 108 (2): 226-232. doi:10.1016/j.ijfoodmicro.2005.12.007 
Sánchez M.X., Fluckey, W.M., Brashears, M.M. \& McKee, S.R. 2002. Microbial profile and antibiotic susceptibility of Campylobacter spp. and Salmonella spp. in broilers processed in airchilled and immersion-chilled environments. Journal of Food Protection 6: 911-1053.

Servicio Nacional de Salud Animal (SENASA). 2013. Decreto $n^{\circ}$ 37548: Reglamento Sanitario y de Inspección Veterinaria de establecimientos de sacrificio y procesadores de aves. San José, Costa Rica.

Solow, B.T, Cloak, O.M. \& Fratamico, P.M. 2003. Effect of temperature on viability of Campylobacter jejuni and Campylobacter coli on raw chicken or pork skin. Journal of Food Protection 11: 1964-2179.

Wesley, I.V., Muraoka, W.T., Trampel, D.W. \& Hurd, H.S. 2005. Effect of Preslaughter Events on Prevalence of Campylobacter jejuni and Campylobacter coli in Market-Weight Turkeys. Applied and Environmental Microbiology 71(6): 2824-2831. doi:10.1128/ AEM.71.6.2824-2831.2005

Whyte, P., Collins, J.D., McGill, K., Monahan, C. \& O'Mahony, H. 2001. The effect of transportation stress on excretion rates of Campylobacter in market-age broilers. Poultry Science 80: 817-820.

Wideman, N., Bailey, M., Bilgili, S.F., Thippareddis, H., Wang, L., Bratchet, C., Sánchez-Plata, M. \& Singh, M. 2015. Evaluating best practices for Campylobacter and Salmonella reduction in poultry processing plants. Poultry Science 00: 1-10.doi:10.3382/ps/pev328

Wilson, I.G. 2002. Salmonella and Campylobacter contamination of raw retail chickens from different producers: a six year survey. Epidemiology and Infection 129 (03): 635-645

Wright, C. 2010. La industria avícola costarricense. Consultado: 21 de febrero del 2013 http/www.wattagnet.com

Yang, H., Li, Y. \& Johnson, M.G. 2001. Survival and death of Salmonella typhimurium and Campylobacter jejuni in processing water and on chicken skin during poultry scalding and chilling. Journal of Food Protection 6: 759-906.

Zumbado L. 2016. Prevalencia y especies de Campylobacter spp. en pollo de engorde, determinados mediante $P C R$, y factores asociados a la contaminación por esta bacteria en tres niveles de la cadena avícola de Costa Rica. (Tesis de Maestría). Universidad Nacional. Heredia, Costa Rica.

Zumbado-Gutiérrez, L., Arévalo-Madrigal, A., Donado-Godoy, M. \& Romero-Zúñiga, J. 2014. Diagnóstico molecular de Campylobacter en la cadena avícola destinada para consumo humano en Costa Rica. Agronomía Mesoamericana, 25(2): 357. http://dx.doi. org/10.15517/am.v25i2.15443 